\title{
Interpretation of zircon coronae textures from metapelitic granulites of the Ivrea-Verbano Zone, northern Italy: two-stage decomposition of Fe-Ti oxides
}

Elizaveta Kovaleva et al.

Correspondence to: Elizaveta Kovaleva (kovalevae@ufs.ac.za)

The copyright of individual parts of the supplement might differ from the CC BY 3.0 License. 


\begin{tabular}{|c|c|c|c|c|c|c|c|c|c|c|c|c|c|c|c|c|}
\hline DataPoint & mineral & $\mathrm{Na}$ & $\mathrm{Si}$ & $\mathrm{Mg}$ & $\mathrm{Al}$ & $\mathrm{Ca}$ & $\mathrm{Mn}$ & $\mathrm{Fe}$ & $\mathrm{Ti}$ & $\mathrm{Cr}$ & $\mathrm{Ni}$ & $\mathrm{K}$ & $\mathrm{Ba}$ & $\mathrm{Sr}$ & $\mathrm{Zn}$ & $\mathrm{Nb}$ \\
\hline $42 / 1$. & Garnet core & -2 & 230 & 245 & 210 & 259 & 312 & 790 & 250 & & & & & & & \\
\hline $43 / 1$. & Garnet rim & 343 & 215 & 241 & 217 & 256 & 313 & 821 & 255 & & & & & & & \\
\hline $44 / 1$. & Garnet rim & 353 & 223 & 242 & 227 & 252 & 308 & 804 & 250 & & & & & & & \\
\hline $45 / 1$ & Garnet rim & -2 & 230 & 248 & 206 & 246 & 323 & 806 & 264 & & & & & & & \\
\hline $46 / 1$. & Garnet rim & 399 & 224 & 235 & 211 & 244 & 311 & 795 & 251 & & & & & & & \\
\hline $47 / 1$. & Bt rim & 404 & 267 & 237 & 222 & 252 & 313 & 676 & 294 & & & 453 & & & & \\
\hline $48 / 1$ & Bt rim & 370 & 260 & 238 & 210 & 239 & 296 & 702 & 294 & & & 440 & & & & \\
\hline $49 / 1$ & Bt core & 388 & 266 & 252 & 220 & 242 & 294 & 604 & 303 & & & 441 & & & & \\
\hline $50 / 1$. & Bt core & 389 & 267 & 239 & 214 & 234 & 290 & 590 & 295 & & & 445 & & & & \\
\hline $51 / 1$. & Bt rim & 406 & 272 & 244 & 219 & 235 & 307 & 679 & 278 & & & 435 & & & & \\
\hline $52 / 1$. & Phyl & 438 & 291 & 261 & 325 & 288 & & 530 & 0 & & & 476 & 996 & -1 & & \\
\hline $53 / 1$. & Phyl & 363 & 293 & 244 & 308 & 321 & & 456 & 356 & & & 530 & 981 & 986 & & \\
\hline $54 / 1$ & Phyl & 482 & 299 & 237 & 319 & 310 & & 528 & 363 & & & 486 & 967 & 0 & & \\
\hline $55 / 1$. & Bt rim & 391 & 257 & 241 & 217 & 232 & 298 & 685 & 293 & & & 396 & & & & \\
\hline $56 / 1$. & Chl & -1 & 247 & 252 & 211 & 227 & 293 & 731 & 274 & & & 296 & & & & \\
\hline $57 / 1$ & Chl & 0 & 260 & 249 & 229 & 228 & 285 & 743 & 284 & & & 309 & & & & \\
\hline $58 / 1$ & IIm & & 191 & 216 & 160 & & 341 & 820 & 349 & 0 & 0 & & & & 663 & 1048 \\
\hline $59 / 1$ & IIm & & 188 & 210 & 161 & & 345 & 789 & 347 & 405 & 419 & & & & -4 & 941 \\
\hline $60 / 1$ & Rut & & 158 & 189 & 102 & & & 314 & 877 & 214 & & & & & & 516 \\
\hline $61 / 1$. & Rut & & 158 & 191 & 104 & & & 328 & 883 & 211 & & & & & & 527 \\
\hline $62 / 1$ & Rut & & 158 & 190 & 103 & & & 340 & 861 & 218 & & & & & & 515 \\
\hline $63 / 1$ & Grt core & 0 & 222 & 246 & 222 & 264 & 308 & 830 & 261 & & & & & & & \\
\hline $64 / 1$. & Grt rim & 376 & 225 & 247 & 203 & 249 & 316 & 835 & 260 & & & & & & & \\
\hline $65 / 1$ & Grt core & 363 & 224 & 251 & 223 & 261 & 315 & 812 & 265 & & & & & & & \\
\hline $66 / 1$ & Grt rim & -1 & 225 & 252 & 207 & 255 & 314 & 833 & 259 & & & & & & & \\
\hline $67 / 1$. & Grt rim & 359 & 225 & 247 & 208 & 231 & 314 & 838 & 250 & & & & & & & \\
\hline $68 / 1$. & Chl & 0 & 246 & 246 & 226 & 232 & 282 & 732 & 280 & & & 302 & & & & \\
\hline $69 / 1$. & Chl & -22 & 254 & 243 & 237 & 239 & 288 & 781 & 264 & & & 303 & & & & \\
\hline $70 / 1$ & Chl & 418 & 260 & 251 & 225 & 238 & 306 & 763 & 270 & & & 304 & & & & \\
\hline $71 / 1$. & Phyl & 391 & 267 & 216 & 218 & 208 & 281 & 541 & 265 & & & 404 & & & & \\
\hline 72 / 1 & Phyl & 347 & 256 & 209 & 224 & 213 & 285 & 466 & 268 & & & 421 & & & & \\
\hline $73 / 1$ & Phyl & 307 & 258 & 205 & 215 & 243 & 296 & 483 & 268 & & & 397 & & & & \\
\hline $74 / 1$ & Chl & 398 & 246 & 251 & 226 & 235 & 298 & 734 & 283 & & & 300 & & & & \\
\hline $75 / 1$ & Chl & 390 & 252 & 236 & 222 & 242 & 284 & 734 & 269 & & & 306 & & & & \\
\hline $76 / 1$. & Phyl & 338 & 261 & 208 & 215 & 227 & 289 & 507 & 279 & & & 406 & & & & \\
\hline $77 / 1$. & $\mathrm{Ilm}$ & & 209 & 247 & 173 & & 383 & 883 & 392 & -1 & 0 & & & & -1 & 1109 \\
\hline $78 / 1$ & Grt rim & -2 & 221 & 242 & 205 & 246 & 315 & 852 & 260 & & & & & & & \\
\hline $79 / 1$ & Grt rim & -2 & 222 & 242 & 215 & 270 & 314 & 878 & 260 & & & & & & & \\
\hline $80 / 1$. & Grt rim & 0 & 222 & 245 & 206 & 259 & 321 & 860 & 0 & & & & & & & \\
\hline $81 / 1$. & Chl & 410 & 250 & 249 & 226 & 225 & 291 & 755 & 284 & & & 307 & 843 & & & \\
\hline $82 / 1$ & Chl & 439 & 253 & 242 & 229 & 238 & 300 & 753 & 281 & & & 297 & 836 & & & \\
\hline $83 / 1$ & Phyl & 371 & 253 & 220 & 224 & 234 & 291 & 569 & 271 & & & 395 & 845 & & & \\
\hline $84 / 1$ & Pheng/mus & 352 & 266 & 203 & 217 & 238 & 0 & 428 & 273 & & & 431 & 909 & & & \\
\hline $85 / 1$. & Pheng/mus & 370 & 258 & 199 & 213 & 240 & 0 & 415 & 265 & & & 419 & 866 & & & \\
\hline $86 / 1$ & Phyl & 366 & 257 & 200 & 216 & 250 & 273 & 487 & 279 & & & 389 & 885 & & & \\
\hline $87 / 1$. & Pheng/mus & 352 & 254 & 207 & 219 & 232 & 281 & 455 & 276 & & & 438 & 882 & & & \\
\hline $88 / 1$ & Grt core & 344 & 222 & 238 & 214 & 251 & 313 & 822 & 254 & & & & & & & \\
\hline $89 / 1$. & Grt core & 362 & 225 & 230 & 214 & 257 & 311 & 837 & 256 & & & & & & & \\
\hline $90 / 1$ & Chl & 0 & 254 & 244 & 235 & 226 & 288 & 732 & 288 & & & 310 & 827 & & & \\
\hline $91 / 1$. & Bt rim & 421 & 256 & 241 & 223 & 243 & 297 & 713 & 305 & & & 391 & 908 & & & \\
\hline $92 / 1$ & Chl & 0 & 251 & 239 & 212 & 228 & 294 & 741 & 270 & & & 299 & 0 & & & \\
\hline $93 / 1$ & Chl & -1 & 246 & 252 & 227 & 239 & 294 & 730 & 276 & & & 314 & 822 & & & \\
\hline $94 / 1$ & Grt rim & -3 & 220 & 240 & 207 & 259 & 300 & 821 & 263 & & & & & & & \\
\hline $95 / 1$ & Grt rim & 369 & 219 & 234 & 209 & 260 & 312 & 824 & 260 & & & & & & & \\
\hline $96 / 1$ & Grt core & 375 & 220 & 243 & 213 & 247 & 303 & 800 & -1 & & & & & & & \\
\hline
\end{tabular}


97/1. Grt core $\quad 344 \quad 223 \quad 237 \quad 197 \quad 266 \quad 316 \quad 778 \quad 254$

$98 / 1 . \quad$ Grt core $\quad 380 \quad 224 \quad 237 \quad 211 \quad 256 \quad 315 \quad 780 \quad 253$

Supplemental Table S1. Detection limits of EMPA analyses in ppm. 


\begin{tabular}{|c|c|c|c|c|}
\hline $\mathrm{Ta}$ & Comment & $x$ & Y & Z \\
\hline & IV12-05_pos01_1 & 12338 & 31291 & -11 \\
\hline & IV12-05_pos01_2 & 12139 & 31101 & -11 \\
\hline & IV12-05_pos01_3 & 12265 & 31074 & -11 \\
\hline & IV12-05_pos01_4 & 12459 & 31134 & -13 \\
\hline & IV12-05_pos01_6 & 12610 & 31094 & -15 \\
\hline & IV12-05_pos01_7 & 12558 & 31116 & -15 \\
\hline & IV12-05_pos01_8 & 12538 & 31188 & -15 \\
\hline & IV12-05_pos01_9 & 12767 & 31243 & -19 \\
\hline & IV12-05_pos01_10 & 12233 & 30928 & -17 \\
\hline & IV12-05_pos01_11 & 12234 & 30961 & -17 \\
\hline & IV12-05_pos01_12 & 12438 & 30987 & -17 \\
\hline & IV12-05_pos01_13 & 12543 & 30983 & -17 \\
\hline & IV12-05_pos01_14 & 12279 & 30999 & -17 \\
\hline & IV12-05_pos01_15 & 12348 & 30856 & -17 \\
\hline & IV12-05_pos01_16 & 12373 & 30891 & -17 \\
\hline & IV12-05_pos01_17 & 12184 & 30842 & -17 \\
\hline 1150 & IV12-05_pos01_18 & 17831 & 22603 & -78 \\
\hline 1155 & IV12-05_pos01_19 & 18042 & 22604 & -78 \\
\hline & IV12-05_pos01_20 & 17932 & 22531 & -78 \\
\hline & IV12-05_pos01_21 & 17947 & 22582 & -78 \\
\hline & IV12-05_pos01_22 & 18004 & 22759 & -78 \\
\hline & IV12-05_pos02_23 & 17724 & 22788 & -73 \\
\hline & IV12-05_pos02_24 & 17782 & 22708 & -73 \\
\hline & IV12-05_pos02_25 & 17861 & 22743 & -73 \\
\hline & IV12-05_pos02_26 & 17879 & 22703 & -73 \\
\hline & IV12-05_pos02_27 & 17865 & 22589 & -77 \\
\hline & IV12-05_pos02_28 & 18133 & 22696 & -82 \\
\hline & IV12-05_pos02_29 & 18227 & 22651 & -85 \\
\hline & IV12-05_pos02_30 & 17792 & 22633 & -82 \\
\hline & IV12-05_pos02_31 & 17829 & 22589 & -82 \\
\hline & IV12-05_pos02_32 & 17936 & 22758 & -82 \\
\hline & IV12-05_pos02_33 & 17947 & 22744 & -82 \\
\hline & IV12-05_pos02_34 & 17835 & 22554 & -82 \\
\hline & IV12-05_pos02_35 & 17835 & 22554 & -82 \\
\hline & IV12-05_pos02_36 & 17729 & 22638 & -82 \\
\hline 1269 & IV12-05_pos03_37 & 16696 & 16242 & -102 \\
\hline & IV12-05_pos03_38 & 16993 & 16635 & -102 \\
\hline & IV12-05_pos03_39 & 16934 & 16603 & -99 \\
\hline & IV12-05_pos03_40 & 16937 & 16448 & -99 \\
\hline & IV12-05_pos03_41 & 16945 & 16232 & -103 \\
\hline & IV12-05_pos03_42 & 16981 & 16233 & -103 \\
\hline & IV12-05_pos03_43 & 16912 & 16276 & -103 \\
\hline & IV12-05_pos03_44 & 16970 & 16277 & -103 \\
\hline & IV12-05_pos03_45 & 16731 & 16234 & -103 \\
\hline & IV12-05_pos03_46 & 17005 & 16257 & -103 \\
\hline & IV12-05_pos03_47 & 16581 & 16500 & -103 \\
\hline & IV12-05_grt1_c & 16811 & 15481 & -100 \\
\hline & IV12-05_grt1_r & 16648 & 15610 & -100 \\
\hline & IV12-05_pos04_48 & 2393 & 23044 & 12 \\
\hline & IV12-05_pos04_49 & 2487 & 23203 & 12 \\
\hline & IV12-05_pos04_50 & 2264 & 23191 & 12 \\
\hline & IV12-05_pos04_51 & 2187 & 22927 & 12 \\
\hline & IV12-05_pos04_52 & 2395 & 23026 & 12 \\
\hline & IV12-05_pos04_53 & 2489 & 23188 & 12 \\
\hline & IV12-05_pos04_54_c & 2684 & 23008 & 12 \\
\hline
\end{tabular}


IV12-05_pos04_55_c IV12-05_pos04_56_c
2658

2878

22820

22833

12

12 